\title{
Ontogênese, estresse e dependência de substâncias psicoativas
}

\author{
Cleopatra da Silva Planeta ${ }^{1 *}$, Fábio Cardoso Cruz', Marcelo Tadeu Marin', Moacyr Luiz \\ Aizenstein'2, Roberto DeLucia ${ }^{2}$
}

\begin{abstract}
${ }^{1}$ Laboratório de Neuropsicofarmacologia, Departamento de Princípios Ativos Naturais e Toxicologia, Faculdade de Ciências Farmacêuticas, Universidade Estadual Paulista, Araraquara, ${ }^{2}$ Laboratório de Psicofarmacologia, Instituto de Ciências Biomédicas, Universidade de São Paulo
\end{abstract}

${ }^{*}$ Correspondência:

C. S. Planeta

Laboratório de

Neuropsicofarmacologia

Faculdade de Ciências Farmacêuticas

Universidade Estadual Paulista -

UNESP

Rodovia Araraquara - Jaú km 01

14801-902 - Araraquara - SP, Brasil

E-mail: cplaneta@fcfar.unesp.br
O estudo da dependência de substâncias psicoativas apresentou grandes avanços conceituais nas últimas décadas. A evolução dos conceitos foi paralela às evidências científicas que têm revelado os aspectos comportamentais e os mecanismos neurais envolvidos nesse fenomeno. Contudo, um grande desafio que permanece na pesquisa sobre a dependência de substâncias psicoativas é a identificação de quais fatores são responsáveis pela transição do uso controlado para o uso compulsivo. Está demonstrado que muitas variáveis interagem para influenciar a probabilidade de que qualquer individuo inicie o uso abusivo de substâncias psicoativas ou se torne dependente. Nos últimos anos, o estresse tem sido destacado como um fator importante na iniciação, manutenção e recaída da utilização de substâncias psicoativas. Neste trabalho analisamos os conceitos e teorias da farmacodependência e as principais evidências comportamentais pré-clínicas que demonstram a relação entre estresse e a vulnerabilidade ao abuso e dependência de psicoestimulantes.
Unitermos

- Estresse

- Dependência

- Psicoestimulantes

- Ontogênese

\section{INTRODUÇÃO}

\section{Conceitos e teorias}

A dependência de substâncias psicoativas é caracterizada por um conjunto de sintomas indicativos de que o indivíduo perdeu o controle do uso da substância e o mantém a despeito das suas conseqüências adversas. Na sua forma extrema, a dependência caracteriza-se pelo uso compulsivo da substância (O’Brien, 2005).

Nos primeiros estudos sistemáticos com o objetivo de entender a farmacodependência predominaram as investi- gações das conseqüências aversivas decorrentes da interrupção do uso das substâncias. Isso se deve ao fato de que os primeiros estudos foram realizados com opióides e etanol, os quais produzem tolerância e síndrome de abstinência marcantes (Jaffe, 1990).

Os sintomas clássicos da síndrome de abstinência como, por exemplo, sudorese, náusea, cãibras, convulsões e taquicardia observadas após a interrupção do uso de opióides ou etanol, são evidentes e podem ser mensurados objetivamente. Assim, a dependência a essas substâncias foi denominada dependência física (Wise, Bozarth, 1987). A dependência física é um estado que resulta das 
adaptações de diferentes circuitos neurais afetados pelas substâncias psicoativas. Essas neuroadaptações manifestam-se como tolerância no decorrer do uso da substância e como síndrome de abstinência quando ocorre a interrupção da sua utilização (O’Brien, 2005).

A tolerância caracteriza-se pela diminuição dos efeitos de uma dose fixa da substância psicoativa no decorrer da administração prolongada, ou ainda, pela necessidade de se aumentar a dose para obtenção dos efeitos iniciais. A tolerância farmacodinâmica resulta da alteração do equilíbrio ou homeostase de circuitos neurais, de tal forma que esses atingem novos pontos de equilíbrio na presença de inibição ou estimulação por uma determinada substância. O processo de alteração da homeostase decorre do fenômeno denominado genericamente neuroadaptação (Jaffe, 1990).

Os mecanismos da neuroadaptação estão relacionados à capacidade das substâncias de induzir alterações na liberação de neurotransmissores, na densidade de receptores ou nos processos de acoplamento e transdução das vias neurais afetadas pelas mesmas (Jaffe, 1990).

Com o desenvolvimento da tolerância, o organismo requer a administração continuada da substância psicoativa para manter as suas funções. Quando ocorre a suspensão abrupta do uso da substância psicoativa, o sistema expressa seu estado de desequilíbrio dando origem à síndrome de abstinência. Com base nessas observações foi proposto que indivíduos dependentes fisicamente manteriam o uso da substância psicoativa para evitar o desconforto da retirada, dando origem assim à teoria do reforço negativo (Jaffe, 1990).

Outros mecanismos, além das neuroadaptações, podem contribuir para o desenvolvimento da tolerância. Assim, a tolerância farmacocinética decorre de modificações nos processos farmacocinéticos, como por exemplo indução enzimática, de tal forma que concentrações menores da substância administrada atingem o seu local de ação (O’Brien, 2005).

A observação de que algumas substâncias psicoativas que produzem dependência, como a cocaína e a anfetamina, não apresentavam a síndrome de abstinência clássica gerou as primeiras tentativas de modificação da teoria do reforço negativo. Assim, em 1965, foi introduzido o termo dependência psíquica, que seria extensivo a todas as classes de substâncias psicoativas, inclusive àquelas que não apresentavam a síndrome de abstinência clássica (Eddy, Halbach, Seevers, 1965).

Contudo, posteriormente, muitos trabalhos demonstraram que todas as substâncias psicoativas que produzem dependência apresentam síndrome de abstinência. Assim, embora, nicotina, cocaína e maconha não produzam a síndrome de abstinência clássica observada após a retirada de opióides ou etanol, todas essas substâncias produzem sinais e sintomas típicos após a interrupção do uso, caracterizando, assim, a síndrome de abstinência (Gawin, Ellinwood, 1986; O’Brien, 2005). Por exemplo, após a retirada abrupta de cocaína, observa-se sonolência, depressão, fadiga e bradicardia. A síndrome de abstinência à maconha é caracterizada por agitação, irritabilidade, insônia, náuseas e distúrbios do sono. Após a interrupção do uso de nicotina podem-se observar irritabilidade, impaciência, aumento do apetite, ganho de peso, bradicardia, ansiedade e dificuldade de concentração (O’Brien, 2005).

Entretanto, mesmo com a demonstração de que todas as substâncias psicoativas apresentam síndrome de abstinência e, portanto, induzem dependência física, a teoria do reforço negativo apresenta várias limitações para explicar a farmacodependência. Por exemplo, o tratamento da síndrome de abstinência é pouco eficaz para tratar a dependência (Wise, Bozarth, 1987). Outra limitação é que a síndrome de abstinência tem duração limitada, entretanto, existe alta tendência de recaída mesmo após vários anos de interrupção do uso da substância psicoativa, quando os sintomas da abstinência não estão mais presentes. Além disso, o início da experimentação de substâncias de abuso ocorre na ausência de síndrome de abstinência (Jaffe et al., 1989; Foltin, Fischman, 1991).

Em 1987, Wise e Bozarth (1987) enfatizaram o valor heurístico da investigação dos elementos comuns subjacentes ao fenômeno da dependência. Dessa forma, o conhecimento relativo a uma substância psicoativa poderia ser estendido às outras, elaborando-se, assim, uma teoria geral para explicar a farmacodependência.

A síndrome de abstinência não estaria incluída entre esses elementos porque, embora todas as substâncias psicoativas que produzem dependência induzam síndrome de abstinência, os sinais e sintomas são específicos para cada substância psicoativa (ou classe de substâncias) e, portanto, devem ser mediados por neuroadaptações de sistemas distintos.

Assim, Wise e Bozarth (1987) propuseram que todas as substâncias psicoativas que induzem dependência têm em comum a propriedade de causar efeitos euforizantes ou prazerosos e, dessa forma, atuariam como reforçadores positivos. A teoria do reforço positivo propõe que a autoadministração da substância psicoativa é mantida por causa do estado que ela produz e não porque ela alivia um estado de desconforto.

O efeito reforçador positivo das substâncias psicoativas tem sido amplamente demonstrado em modelos experimentais baseados nos princípios do condicionamento clássico (preferência condicionada por lugar) ou operante (auto-administração). 
Neste sentido, demonstrou-se que nicotina, cocaína, anfetamina, fencanfamina, delta-9-THC, opióides e etanol induzem preferência condicionada por lugar e autoadminstração (Spyraki et al., 1982; Shippenberg et al., 1989; Lepore et al., 1995; Horan et al., 1997; Tanda, Munzar, Goldberg, 2000; Bozarth, 1990; Planeta, Aizenstein, DeLucia, 1995a).

Wise e Bozarth (1987) sugeriram, também, que o efeito reforçador positivo das substâncias psicoativas é decorrente da ativação de um substrato neurobiológico comum - o sistema dopaminérgico mesocorticolímbico. Os principais componentes desse circuito são a área tegmental ventral (sítio de corpos celulares de neurônios dopaminérgicos) e suas projeções para regiões do sistema límbico incluindo o núcleo acumbens, o tubérculo olfativo, a amígdala e o córtex frontal e límbico (Koob, 1992; Koob, Le Moal, 2001). Muitas evidências comprovam esta hipótese. Assim, estudos de microdiálise demonstraram que a administração de cocaína, anfetamina, morfina (Pontiere et al., 1995), fencanfamina (Kuczenski, Segal, Aizenstein, 1991) etanol (Kiianmaa et al., 1995), delta-9-THC (Tanda et al., 1997) e nicotina (Pontiere, Tanda, Chiara, 1996) aumentam a liberação de dopamina no núcleo acumbens.

O efeito reforçador positivo, ou seja, a sensação de prazer causada pela substância psicoativa, explica a experimentação e o seu uso ocasional. Contudo, essa teoria não explica as principais características da dependência como ela é conceituada atualmente: a perda do controle do uso da substância e seu uso a despeito das conseqüências adversas (World Health Organization 1992; O’Brien, 2005).

Embora as substâncias psicoativas possam produzir estados afetivos de prazer extremamente intensos é difícil que essa propriedade isoladamente seja suficiente para explicar a dependência. Além disso, as conseqüências negativas do uso continuado dessas substâncias freqüentemente se sobrepõem ao prazer obtido com as mesmas. De fato, para a maioria dos indivíduos dependentes, as conseqüências negativas do uso da substância psicoativa, como por exemplo, prejuízos à saúde, das atividades profissionais e sociais são enormes se comparados aos efeitos prazerosos proporcionados pela substância (Robinson, Berridge, 1993).

A fim de preencher a lacuna deixada pela teoria do reforço positivo, Robinson e Berridge (1993) propuseram que o uso compulsivo das substâncias psicoativas resultaria de processos neuroadaptativos causados pelo uso repetido das mesmas. É amplamente conhecido o fato de que os efeitos das substâncias psicoativas se modificam no decorrer do uso prolongado. Essas alterações expressam-se como tolerância ou sensibilização comportamental.

A tolerância resulta de adaptações de vias neurais que medeiam efeitos específicos da substância psicoativa e dão origem à síndrome de abstinência na sua retirada. Entretanto, como relatado ateriormente, a tolerância e a síndrome de abstinência são específicas para cada classe de substância e não explicam a característica comum da farmacodependência, ou seja, a perda do controle e o uso compulsivo. De fato, tolerância sem compulsão pode ser observada em pacientes que utilizam morfina para tratamento da dor (Jage, 2005) ou benzodiazepínicos para o tratamento de transtornos da ansiedade (O'Brien, 2005). Contudo, o uso compulsivo e múltiplas recaídas podem ocorrer em dependentes que não apresentam sintomas significativos de retirada. Assim, embora a tolerância e conseqüente síndrome de retirada sejam importantes para a manutenção do uso da substância elas não contribuem para a compreensão dos mecanismos neurais da farmacodependência (Hyman, Malenka, Nestler, 2006).

Vários estudos demonstram que a administração repetida de todas as substâncias que produzem dependência causa aumento progressivo da atividade locomotora e esse fenômeno é denominado sensibilização comportamental (Post, Contel, 1983; Robinson, Becker, 1986). A sensibilização comportamental foi descrita para a cocaína (Miserendino, Nestler, 1995), anfetamina (Robinson, Becker, 1986; Aizenstein, Segal, Kuczenski, 1990; Vezina, Queen, 2000); fencanfamina (Aizenstein, Segal, Kuczenski, 1990; Planeta, Aizenstein, DeLucia, 1995b) morfina (Kalivas, Duffy, 1987), etanol (Phillips, Roberbs, Lessov, 1997), nicotina (Domino, 2001; Shim et al., 2001) e delta-9-THC (Cadoni et al., 2001).

A sensibilização comportamental tem sido descrita em animais de experimentação desde 1932 e em humanos desde 1950 e foi tradicionalmente utilizada como modelo experimental de psicose "anfetamínica" ou "cocaínica", que pode ocorrer em humanos após o uso prolongado de altas doses dessas substâncias psicoativas (Segal, Janowsky, 1978).

A sensibilização comportamental resulta de adaptações neuroquímicas e moleculares do sistema dopaminérgico e tem sido proposta como um mecanismo subjacente a alguns aspectos da farmacodepedência, principalmente aqueles relacionados ao uso compulsivo da substância (Robinson, Becker, 1986; Nestler, Hyman, Robert, 2001).

De acordo com Robinson e Berridge (1993, 2000, 2001), além de mediar a sensação subjetiva de prazer, a dopamina regularia também o impulso motivacional e a atenção a estímulos relevantes, incluindo os estímulos reforçadores. O impulso motivacional pode ser descrito em termos de "querer" enquanto a avaliação hedônica em termos de "gostar". Assim, parece que o "gostar" pode ser 
dissociado do "querer" e que a dopamina pode influenciar esses parâmetros de formas diferentes.

Segundo Robinson e Berridge (1993, 2000, 2001), as substâncias psicoativas produziriam sensibilização do sistema dopaminérgico e isto tornaria os estímulos (uso da substância psicoativa e comportamentos relacionados) altamente relevantes, atrativos e desejados. Assim, com o uso repetido, a substância psicoativa e os estímulos associados tornam-se progressivamente mais atrativos e capazes de controlar o comportamento. Com o uso prolongado, o "querer" evolui para o desejo obsessivo ("fissura"), que se manifesta pelo comportamento de compulsão pela substância.

Covington e Miczek (2001) utilizaram o modelo de auto-administração com acesso contínuo (FR1) à substância psicoativa por 24 horas ("binge"), para avaliar a relação entre sensibilização comportamental e a perda do controle do uso de cocaína, compatibilizando, assim, o paradigma experimental com o conceito atual de dependência e a teoria proposta por Robinson e Berridge (1993).

Covington e Miczek (2001) demonstraram que animais controle (não-sensibilizados) cessam a auto-administração de cocaína após 16 horas ou após o acúmulo de $120 \mathrm{mg} / \mathrm{kg}$ de cocaína. Por outro lado, animais sensibilizados, pela exposição repetida a estresse ou morfina, mantêm a auto-administração por períodos mais prolongados e acumulam quantidades maiores de cocaína. Cabe ressaltar, ainda, que a sensibilização não modificou os outros parâmetros da auto-administração de cocaína (aquisição, curva doseresposta e ponto de ruptura) quando o acesso foi limitado a períodos de 1 a 6 horas, sugerindo que o efeito reforçador da cocaína não foi modificado pela sensibilização.

Assim, os autores sugerem que o aumento da administração de cocaína durante o "binge" observado em animais sensibilizados caracteriza a perda do controle da autoadministração da substância psicoativa, o que poderia ser correlacionado com o uso compulsivo em humanos. Com base nessa observação, Covington e Miczek (2001) sugerem que a sensibilização levaria ao uso compulsivo das substâncias psicoativas.

A teoria da sensibilização postulada por Robinson e Berridge (1993) está de acordo com a hipótese de que o uso compulsivo das substâncias psicoativas envolve o processo de aprendizado associativo (Hyman, Malenka, Nestler, 2006).

Outro aspecto da farmacodependência que tem recebido especial atenção nos últimos anos é o fenômeno da recaída. A recaída é freqüentemente desencadeada por uma única exposição à substância ou a estímulos ambientais e objetos a ela associados. Outro fator importante que pode precipitar a recaída é a exposição a situações aversivas ou estresse. A "fissura", e conseqüente recaída, é um dos as- pectos que mais dificultam o tratamento da dependência à cocaína e de outras substâncias psicoativas. A "fissura" pode ser duradoura e dessa forma a recaída ao uso da substância pode ocorrer após vários anos de abstinência (Dackis, O’Brien, 2003; Weiss, 2005).

\section{Fatores que interferem no abuso e dependência de substâncias psicoativas}

Considerando-se as teorias descritas anteriormente, poderíamos supor que a mera exposição à substância psicoativa seria o fator de risco crítico para o desenvolvimento da dependência. Entretanto, estudos epidemiológicos mostram que muitos indivíduos experimentam vários tipos de substâncias psicoativas por períodos variáveis de tempo, mas somente alguns desenvolvem a farmacodependência. Isso indica que fatores adicionais àqueles relacionados à interação entre a substância psicoativa e o organismo influenciam a progressão para o uso compulsivo (O'Brien et al., 1986).

Muitas variáveis interagem para influenciar a probabilidade de que qualquer pessoa inicie o uso abusivo de substâncias psicoativas ou se torne dependente. Essas variáveis podem ser agrupadas em três categorias: a) substância psicoativa ; b) indivíduo e c) ambiente (O’Brien, 2005).

Em relação às substâncias podem-se citar a disponibilidade, o custo e a via de administração como fatores importantes que podem influenciar o desenvolvimento da dependência (O’Brien, 2005).

Com relação aos indivíduos, a variação interindividual nos efeitos das substâncias psicoativas é um fato bastante comum. Diferenças genéticas nas enzimas relacionadas aos processos de biotransformação e na resposta dos receptores às substâncias psicoativas podem contribuir para os diferentes graus de euforia e reforço positivo obtidos por indivíduos diferentes utilizando a mesma substância psicoativa (O’Brien, 2005). Outro fator importante é a fase da vida. Freqüentemente o uso de substâncias psicoativas inicia-se na adolescência. A adolescência é o período de transição gradual entre a infância e a maturidade. A puberdade é uma das várias alterações ontogênicas que ocorre na adolescência e é caracterizada pelas mudanças fisiológicas e neuroendócrinas, que marcam a maturação sexual. Não há delimitação exata do período da adolescência em humanos, uma vez que nenhum evento marcante sinaliza seu início ou final. Além disso, a duração da adolescência pode ser influenciada por fatores sócioculturais, econômicos e nutricionais, entre outros. O período entre 12 e 18 anos de idade é o mais comumente citado na literatura como adolescência em humanos (Spear, 2000). 
Adolescentes de várias espécies exibem comportamentos típicos desse período que incluem o aumento da interação social com seus pares, busca de novidades e sensações e o comportamento de risco (Spear, 2000). Estes comportamentos podem representar adaptações ontogênicas, que possibilitam a aquisição das habilidades necessárias para atingir a independência da idade adulta. Entretanto, o comportamento de risco traz também alguns aspectos negativos, entre esses podem-se citar o alto risco de gravidez não desejada, infecções por HIV e a experimentação de substâncias psicoativas (Spear, 2000).

Levantamento realizado pelo Centro Brasileiro de Informações sobre Drogas Psicotrópicas (Galduróz et al., 2005) revela que 22,6\% dos estudantes de Ensino Fundamental e Médio das capitais brasileiras já haviam experimentado algum tipo de substância psicotrópica, excluindose álcool e tabaco. O uso na vida de cocaína e crack atingiu $2,7 \%$ entre esses estudantes. Vale ressaltar, ainda, que dos 22,6\% que utilizaram essas substâncias, $65 \%$ encontram-se na faixa etária entre 10-18 anos.

Por fim, fatores ambientais e sociais têm um papel muito importante no abuso das drogas e desenvolvimento da dependência às mesmas. Para alguns indivíduos, jovens principalmente, o uso de substâncias psicoativas pode significar uma forma de protesto contra a autoridade ou pode estar relacionado à ausência de outras fontes de obtenção de prazer (O’Brien, 2005).

Nos últimos anos, o estresse tem sido destacado como um fator importante na iniciação e manutenção do uso da substância psicoativa, assim como na recaída à sua utilização (Gawin, 1991; Gordon, 2002; Goeders, 2002, 2003; Sinhá, 2001; Weiss, 2005).

Vários estudos clínicos demonstram que indivíduos dependentes freqüentemente citam a exposição a estímulos aversivos e estados negativos de humor como justificativa para o início, manutenção e recaída ao uso das substâncias psicoativas (Bradley et al., 1989; Wallace, 1989). Sinha, Catapano, O'Malley (1999) relatam que a exposição a estresse ou simplesmente a apresentação de imagens relacionadas a situações de estresse aumentam significativamente o desejo por cocaína e, dessa forma, o estresse poderia ser um fator desencadeador da recaída.

\section{Estresse e dependência}

O termo estresse pode ser definido como um processo que envolve a percepção, interpretação, resposta e adaptação a eventos aversivos, ameaçadores ou desafiadores (Selye, 1975). A resposta do organismo ao estresse resulta na ativação do eixo hipotálamo-pituitária-adrenal (HPA) e do sistema nervoso simpático.
A primeira etapa da ativação do eixo HPA é a liberação do hormônio liberador de corticotrofina ( $\mathrm{CRH})$, um peptídeo constituído por uma cadeia simples de 41 aminoácidos. Embora o CRH esteja distribuído em várias regiões do sistema nervoso central, a liberação de CRH pelos neurônios da divisão parvocelular do núcleo paraventricular do hipotálamo, que se projetam para a zona externa da eminência média, é o evento responsável pela ativação do eixo HPA. Estes neurônios liberam o CRH na circulação portal adeno-hipofisária num ritmo circadiano ou em resposta à estimulação neuronal (Akil, Morano, 1995).

A interação do CRH com seus receptores localizados na pituitária anterior resulta na síntese de proopiomelanocortina (POMC), uma proteína precursora, que sofre clivagem proteolítica e dá origem a vários peptídeos biologicamente ativos, entre esses o hormônio adrenocorticotrófico (ACTH). O ACTH difunde-se pela circulação sistêmica até atingir as glândulas adrenais. O ACTH interage com seus receptores no córtex da adrenal estimulando a síntese e secreção de adrenocorticosteróides, principalmente os glicocorticóides, cortisol em humanos e corticosterona em roedores (Akil, Morano, 1995). Como decorrência da ativação do sistema nervoso simpático observa-se aumento da freqüência cardíaca, aumento da glicemia, dilatação das pupilas e estimulação da respiração. Estas mudanças fisiológicas preparam o organismo para escapar ou enfrentar o estímulo estressor e para manter a homeostase.

\section{Estresse e efeitos reforçadores da cocaína}

O efeito reforçador positivo das substâncias de abuso tem sido amplamente demonstrado no modelo da autoadministração intravenosa de substâncias psicoativas. Nesse modelo os animais são treinados para emitir uma resposta operante (pressionar uma barra, por exemplo), o que produz a injeção contingente da substância através de um cateter implantado por via intravenosa. Durante a aquisição do comportamento de auto-administração, o animal entra em contato com a cocaína e identifica seus efeitos reforçadores pela primeira vez. Nessa fase o animal aprende a expressar a resposta que produz a administração de cocaína. Estímulos ambientais que facilitam a aquisição da auto-administração são considerados eventos que aumentam a vulnerabilidade ou propensão para o abuso de substâncias psicoativas.

Vários trabalhos demonstraram que a aquisição da auto-administração de anfetamina e cocaína é facilitada pela exposição aguda a diferentes tipos de estresse como "tail pinch" (Piazza et al., 1990) e estresse social (Haney 
et al., 1995; Tidey, Miczek, 1997). Dessa forma, a exposição ao estresse parece aumentar o efeito reforçador da cocaína e, conseqüentemente, a vulnerabilidade ao abuso da substância e desenvolvimento de dependência.

\section{Estresse e sensibilização comportamental}

Vários estudos pré-clínicos demonstram que a exposição repetida a diferentes tipos de estresse, como choque nas patas, imobilização, natação forçada ou estresse social, provocam aumento da resposta locomotora à administração subseqüente de anfetamina ou cocaína caracterizando, assim, a sensibilização cruzada entre estresse e psicoestimulantes (Badiani, Simona, Puglisi-Allegra, 1992; Miczek et al., 1999; Antelman et al., 1980; Deroche et al., 1995; Reid et al., 1998; Haile, Grandpre, Kosten, 2001; Araujo et al., 2003). Nosso laboratório investigou sensibilização cruzada entre estresse e cocaína em ratos adultos e adolescentes. Os animais foram expostos a dois tipos de estresse repetido: previsível, que consistiu de 1 hora de imobilização diária ou imprevisível, no qual o animal foi exposto a diferentes tipos de estresse (por exemplo, natação forçada, imobilização, frio) em ambos os protocolos a duração da exposição foi de 10 dias. Após a exposição ao estresse os animais receberam administração aguda de cocaína $(10,0 \mathrm{mg} / \mathrm{kg})$ e tiveram a atividade locomotora registrada por 60 minutos. Nossos resultados demonstraram a sensibilização cruzada entre estresse e cocaína nos animais adolescentes e adultos. Contudo, enquanto nos adolescentes a sensibilização foi observada após a exposição a ambos os tipos de estresse repetido, nos adultos a sensibilização ocorreu apenas após a exposição a estresse previsível (Araújo et al., 2003; Lepsch et al., 2005). Esses resultados sugerem que a natureza e previsibilidade do estímulo aversivo são fatores importantes quando se considera a influência do estresse sobre os efeitos comportamentais dos psicoestimulantes. Além disso, animais adolescentes parecem ser mais vulneráveis aos efeitos do estresse.

Como ressaltado anteriormente, muitas pessoas fazem uso experimental de cocaína, contudo, apenas uma pequena porcentagem desses usuários desenvolve o padrão de uso compulsivo dessa substância (Warner et al., 1995). Nessa pequena, mas significativa porcentagem da população, o autocontrole do consumo de cocaína deteriora-se em decorrência do seu uso repetido. Um desafio para clínicos e pesquisadores é a identificação dos riscos associados à perda do controle do uso de cocaína (Gawin, 1991). Existem evidências de que a exposição repetida ao estresse poderia ser um dos fatores de risco.

De forma semelhante aos seres humanos, camundon- gos, ratos e primatas não humanos adquirem o comportamento de auto-administração intravenosa de cocaína, que inicialmente se apresenta de forma controlada. Esse controle ocorre quando o acesso à cocaína é limitado em poucas horas diárias. A auto-administração estável de cocaína persiste enquanto o acesso limitado é permitido (Pickens, Thompson, 1968). No procedimento de auto-administração quando a cocaína é disponibilizada continuamente, ela se torna o estímulo controlador e padrões compulsivos de comportamento de auto-administração se desenvolvem em alguns indivíduos, geralmente resultando em conseqüências mórbidas para o animal (Bozarth, Wise, 1985; Johanson, Balster, Bonese, 1976). Tornatzky e Miczek (2000) demonstraram que a exposição a estresse social pode aumentar significativamente a velocidade e duração da auto-administração intravenosa de cocaína em períodos de acesso contínuo de 24 horas.

Recentemente, Convigton et al. (2005) expuseram ratos a estresse social a cada 72 horas durante 10 dias. Sessenta dias após a última sessão de estresse a atividade locomotora dos animais foi avaliada após a injeção aguda de cocaína, revelando a presença de sensibilização cruzada. Os autores demonstram, ainda, que os animais sensibilizados pelo estresse auto-administravam quantidades significativamente maiores de cocaína durante uma sessão de 24 horas de acesso contínuo à substância. Assim, o estresse parece ser um fator importante para a perda do controle da auto-administração em roedores.

\section{Estresse e ontogênese}

As conseqüências da exposição ao estresse podem ser duradouras. Muitas evidências sugerem que, em humanos, a exposição a estresse (por exemplo abuso sexual ou negligência) em fases precoces da vida está associada ao desenvolvimento de transtornos comportamentais, entre estes o abuso e dependência de substâncias psicoativas (Thadani, 2002).

Modelos experimentais em roedores demonstraram que manipulações na fase perinatal podem alterar permanentemente vários parâmetros relacionados ao comportamento de abuso de substâncias psicoativas. A separação materna é um desses procedimentos experimentais. Neste procedimento as ninhadas são afastadas das mães por determinados períodos de tempo (15 minutos a 6 horas) no decorrer das duas primeiras semanas de vida. A separação causa alterações comportamentais e neuroquímicas profundas nos neonatos, que podem perdurar até a idade adulta (Moffett et al., 2007).

Recentemente, avaliamos as conseqüências da separação materna sobre o efeito psicomotor da cocaína em 
ratos adolescentes e adultos (Marin, Planeta, 2004). Nosso protocolo consistiu da separação da ninhada da mãe por 5 horas diárias entre os dias pós-natal 2 e 6 . Após o procedimento de separação os animais foram desmamados aos 21 dias de idade e a partir daí foram alojados em grupos de 4 animais em gaiolas-viveiros sem nenhuma manipulação experimental. Na adolescência (dia pós-natal 35 - 37) e na idade adulta (dia pós-natal 70 - 75) os animais receberam injeção aguda de cocaína $(10,0 \mathrm{mg} / \mathrm{kg})$ e tiveram a atividade locomotora avaliada por 60 minutos. Nossos resultados demonstraram que animais adolescentes expostos à separação materna apresentavam maior atividade locomotora em resposta à cocaína quando comparado aos animais que permaneceram com as mães. Esse resultado caracteriza a presença de sensibilização comportamental. Esse efeito não foi observado quando os animais, também submetidos à separação materna, receberam cocaína na idade adulta. Dessa forma, o estresse neonatal tem efeito duradouro, mas transitório, sobre o efeito psicomotor da cocaína. Entretanto, é importante ressaltar que a presença de sensibilização na adolescência é altamente relevante, uma vez que indica que perturbações neonatais podem aumentar a vulnerabilidade ao desenvolvimento da dependência de cocaína nessa fase do desenvolvimento.

Moffett et al. (2006) avaliaram os efeitos da separação materna na auto-administração de cocaína. Esses autores demonstraram que ratos adultos separados das mães por 6 horas entre os dias pós-natal 2 a 15 adquiriram autoadministração de cocaína em doses menores do que aquelas necessárias para produzir a aquisição em ratos não expostos ao estresse.

Outro modelo de estresse perinatal é o isolamento do neonato. A diferença entre esse modelo e a separação materna é que no último o animal é separado da mãe, mas permanece em contato com a ninhada. Vários trabalhos demonstram que a auto-administração de cocaína pode ser alterada pelo isolamento neonatal. Assim, o isolamento do neonato por $1 \mathrm{~h}$ entre os dias pós-natal 2 e 6 facilitou a aquisição e manutenção do comportamento de auto-administração dessa substância (Kosten et al., 2006; 2004; 2000; Zhang et al., 2005).

\section{Estresse e recaída}

Nas décadas de 1970 e 1980 foram propostos os primeiros modelos pré-clínicos de recaída (Strech, Gerber, Wood, 1971; Wit, Stewart, 1981). Esses são denominados "procedimentos de reinstalação" e têm contribuído significativamente para a compreensão dos mecanismos neurais da recaída.

Os dois modelos de "reinstalação" se baseiam nos princípios do condicionamento operante (auto-administração) ou clássico (preferência condicionada por lugar (PCL).

No primeiro, os animais são inicialmente treinados para auto-administrar uma substância psicoativa, cocaína, por exemplo, pela via intravenosa. Em seguida, procede-se à extinção do comportamento expondo-se o animal à barra sem que o seu pressionar forneça a injeção de cocaína. Após várias exposições o animal para de responder o que caracteriza a extinção da auto-administração. No segundo, os animais inicialmente são treinados para adquirir a PCL. Em seguida, a resposta condicionada é extinta permitindo que o animal explore ambos os compartimentos da caixa de condicionamento sem a administração da substância reforçadora. Após a extinção a capacidade de diferentes estímulos em reinstalar o comportamento extinto é avaliada. A reinstalação da auto-administração ou PCL pelo estímulo sugere que este pode desencadear a recaída em seres humanos.

Ambos os procedimentos demonstram que, de forma semelhante ao observado em humanos, a re-exposição à substância psicoativa ou estímulos a ela associados pode reinstalar a auto-administração ou PCL (Lu et al., 2003; Mueller, Stewart, 2000).

Da mesma forma, a exposição a estresse é capaz de reinstalar auto-administração e PCL induzidas pela cocaína (Capriles et al., 2003; McFarland et al., 2004; Kreibich, Blendy, 2004; Sanchez, Sorg, 2001; Lu et al., 2003). Resultados do nosso laboratório demonstraram que, como observado em ratos adultos, a exposição ao estresse de imobilização reinstala a PCL induzida por anfetamina em animais adolescentes (Cruz et al., 2006).

\section{CONCLUSÃO}

A abordagem biológica da farmacodependência pode modificar profundamente a relação sociedade - dependente e, conseqüentemente, as estratégias de prevenção e tratamento.

A análise dos fatores relacionados à vulnerabilidade ao abuso de substâncias psicoativas e farmacodependência não pode ser restrita apenas às condições imediatas que cercam o indivíduo. $\mathrm{O}$ fato de que a exposição a estresse produz alterações duradouras no sistema nervoso central e isso altera a resposta às substâncias no decorrer da vida é de extrema relevância para enfatizar a necessidade de que medidas educativas de prevenção se iniciem desde as fases mais precoces da vida.

Cabe, portanto, à comunidade científica introduzir os novos conhecimentos à sociedade, para que a dimensão biológica seja incorporada aos aspectos sociais e criminais 
que hoje predominam nas políticas de controle do uso das substâncias psicoativas.

\section{ABSTRACT}

\section{Ortogenesis, stress and psychoactive substances addiction}

The investigation of the mechanisms of drug abuse and addiction showed great advances in the last decades. New concepts emerged from the scientific evidences on behavioral and neural aspects of this phenomenon. However, the biggest challenge for the future is the identification of which risk factors are implicated in the transition from controlled to compulsive drug use. Stress has been pointed as an important factor related to initiation, maintenance and relapse to drug use. In the present paper we discuss the concepts and theories of drug addiction, and the main behavioral pre-clinical evidences showing the relationship between stress and psychostimulant addiction.

UNITERMS: Stress. Addiction. Psychostimulants. Ortogenesis.

\section{REFERÊNCIAS BIBLIOGRÁFICAS}

AIZENSTEIN, M. L.; SEGAL, D. S.; KUCZENSKI, R. Repeated amphetamine and fencamfamine: sensitization and reciprocal cross-sensitization. Neuropsychopharmacology, London, v. 3, p. 283-290, 1990.

AKIL, H. A.; MORANO, M. I. Stress. In: BLOOM, F. E; KUPFER, D. J. (Eds.). Psychopharmacology: the fourth generation of progress. New York: Raven, 1995. p. 773-785.

ANTELMAN, S. M.; EICHLER, A. J.; BLACK, C. A.; KOCAN, D. Interchangeability of stress and amphetamine in sensitization. Science, Washington, v.207, p.329-331, 1980 .

ARAUJO, A. P. N.; DeLUCIA, R.; SCAVONE, C.; PLANETA, C. S. Repeated predictable or unpredictable stress: effects on cocaine - induced locomotion and cyclic AMP-dependent protein kinase activity. Behav. Brain Res., Amsterdam, v.139, p.75 - 81, 2003.

BADIANI, H.; SIMONA, C.; PUGLISI-ALLEGRA, S. Chronic stress induces strain-dependent sensitization to the behavioral effects of amphetamine in the mouse. Pharmacol. Biochem. Behav., New York, v.43, p.53-60, 1992.
BOZARTH, M. A. Evidence for the rewarding effects of ethanol using the conditioned place preference method. Pharmacol. Biochem. Behav., New York, v.35, p.485487, 1990.

BOZARTH, M. A.; WISE, R. A. Toxicity associated with long-term intravenous heroin and cocaine self administration. JAMA, Chicago, v.254, p.81-83, 1985.

BRADLEY, B. P.; PHILLIPS, G.; GREEN, L.; GOSSOP, M. Circumstances surrounding the initial lapse to opiate use following de detoxification. Br. J. Psychiatry, London, v.154, p.354-359, 1989.

CADONI, C.; PISANU, A. SOLINAS, M.; ACQUAS, E.; DI CHIARA, G. Behavioral sensitization after exposure to delta-9-tetrahydrocannabinol and cross-sensitization with morphine. Psychopharmacology, Berlin, v.158, p.259266, 2001.

CAPRILES, N.; RODAROS, D.; SORGE, R. E.; STEWART, J. A role for the prefrontal cortex in stressand cocaine-induced reinstatement of cocaine seeking in rats. Psychopharmacology, Berlin, v.168, p.66-74, 2003.

COVINGTON $3^{\text {rd }}$, H. E.; KIKUSUI, T.; GOODHUE, J.; NIKULINA, E. M.; HAMMER JR, R. P. MICZEK, K. A. Brief social defeat stress: Long lasting effects on cocaine taking during a binge and Zif268 mRNA expression in the amygdala and prefrontal cortex. Neuropsychopharmacol., London, v.30, p.310-321, 2005.

COVINGTON $3^{\text {rd }}$, H. E.; MICZEK, K. A. Repeated social defeat stress or morphine: effects on behavioral sensitization and IV cocaine self-administration "binges". Psychopharmacology, Berlin, v.158, p.388-98, 2001.

CRUZ, F. C.; LEÃO, R. M. ; MARIN, M. T. ; SILVA, R. F. P. ; PLANETA, C. S. Reinstalação da preferência condicionada por lugar à anfetamina induzida pelo estresse em ratos adolescentes. In: REUNIÃO ANUAL DA FEDERAÇÃO DE SOCIEDADES DE BIOLOGIA EXPERIMENTAL, 21., 2006. Resumos. Águas de Lindóia: FeSBE, 2006. CD-ROM.

DACKIS, C.; O'BRIEN, C. Glutamatergic agentes for cocaine dependence. Ann. N. Y. Acad. Sci., New York, v.1003, p.328-345, 2003. 
DEROCHE, V.; MARINELLI, M.; MACCARI, S.; LE MOAL, M.; SIMON, H.; PIAZZA, P. V. Stress-induced sensitization and glucocorticoids. I. Sensitization of dopamine-dependent locomotor effects of amphetamine and morphine depends on stress-induced corticosterone secretion. J. Neurosci., Washington, v.15, p.7181-7188, 1995.

DOMINO, E. F. Nicotine-induced behavioral locomotor sensitization. Prog. Neuro-psychopharmacol. Biol. Psych., Oxford, v.25, p.59-71, 2001

EDDY, N. B.; HALBACH, H.; SEEVERS, M. H. Drug dependence: its significance and characteristics. Bull. WHO, Geneva, v.32, p.721-733, 1965.

FOLTIN, R. W.; FISCHMAN, M. W. Assessment of abuse liability of stimulant drugs in humans: a methodological survey. Drug Alcohol Depend., Limerick, v.28, p.3-48, 1991.

GALDURÓZ, J. C. F.; NOTO, A. R.; FONSECA, A. R.; CARLINI, E. A. V Levantamento nacional sobre o consumo de drogas psicotrópicas entre estudantes do ensino fundamental e médio da rede pública de ensino nas 27 capitais brasileiras. São Paulo: CEBRID -Centro Brasileiro de Informações Sobre drogas Psicotrópicas, 2005. 398 p.

GAWIN, F. H. Cocaine addiction: psychology and neurophysiology. Science, Washington, v.251, p.15801586, 1991.

GAWIN, F. H.; ELLINWOOD, E. H. Abstinence symptomatology and psychiatry diagnoses in cocaine abusers. Arch. Gen. Psych., Chicago, v.43, p.107-113, 1986.

GOEDERS, N. E. The HPA axis and cocaine reinforcement. Psychoneuroendocrinology, Oxford, v.27, p.13-33, 2002.

GOEDERS, N. E. The impact of stress on addiction. Eur Neuropsychopharmacology, Amsterdam, v.13, p. 435441, 2003.

GORDON, H. W. Early environmental stress and biological vulnerability to drug abuse. Psychoneuroendocrinology, Oxford, v.27, p.115-126, 2002.
HAILE, C. N; GRANDPRE, T; KOSTEN T. A. Chronic unpredictable stress, but not chronic predictable stress, enhances the sensitivity to the behavioral effects of cocaine in rats. Psychopharmacology, Berlin, v.154, p.213-20, 2001.

HANEY, M.; MACCARI, S.; LE MOAL, M.; SIMON, H.; PIAZZA, P. V. Social stress increases the acquisition of cocaine self-administration in male and female rats. Brain Res., Amsterdam, v.698, p.46-52, 1995.

HORAN, B.; SMITH, M.; GARDNER, E. L.; LEPORE, M.; ASHBY, C. R. (-)-Nicotine produces conditioned place preference in Lewis, but not Fischer 344 rats. Synapse, Paris, v.26, p.93-94, 1997.

HYMAN, S. E.; MALENKA, R. C.; NESTLER, E. J. Neural mechanisms of addiction: the role of reward-related learning and memory. Annu. Rev. Neurosci., Palo Alto, v.29, p.565-598, 2006.

JAFFE, J. H. Drug addiction and drug abuse. In: GILMAN, A. G.; RALL, T. W.; NIES, A.S.; TAYLOR, P. (Eds.). Goodman and Gilman's the pharmacological basis of therapeutics. 8.ed. New York: Pergamon Press, 1990. p. 523-573.

JAFFE, J. H.; CASCELLA, N. G.; KUMOR, K. M.; SHERER, M. A. Cocaine-induced cocaine craving, Psychopharmacology, Berlin, v.97, p.59-64, 1989.

JAGE, J. Opioid tolerance and dependence - do they matter? Eur. J. Pain, London, v.9, p.157-162, 2005.

JOHANSON, C. E.; BALSTER, R. L.; BONESE, K. Self administration of psychomotor stimulant drugs: the effects of unlimited access. Pharmacol. Biochem. Behav., New York, v.4, p.45-51, 1976.

KALIVAS, P. W.; DUFFY, P. Sensitization to repeated morphine injection in the rat: possible involvement of A10 dopamine neurons. J. Pharmacol. Exp. Ther., Bethesda, v.241, p. 204-212, 1987.

KIIANMAA, K.; NURMI, M.; NYKANEN, I.; SINCLAIR, J. D. Effects of ethanol on extracellular dopamine in the nucleus accumbens of alcohol-preferring AA and alcoholavoiding ANA rats. Pharmacol. Biochem. Behav., New York, v.52, p.29-34, 1995. 
KOOB, G. F. Neural mechanisms of drug reinforcement. Ann. N. Y. Acad. Sci., New York, v. 654, p. 171-191. 1992.

KOOB, G. F.; LE MOAL, M. Drug addiction, dysregulation of reward, and allostasis. Neuropsychopharmacology, London, v. 24, p.97-129, 2001.

KOSTEN, T. A.; MISERENDINO, M. J.; KEHOE, P. Enhanced acquisition of cocaine self-administration in adult rats with neonatal isolation stress experience. Brain Res., Amsterdam, v. 875, p.44-50, 2000.

KOSTEN, T. A.; SANCHEZ, H.; ZHANG, X. Y.; KEHOE, $P$. Neonatal isolation enhances acquisition of cocaine self - administration and food responding in female rats. Behav. Brain Res., Amsterdam, v.151, p.137-149, 2004.

KOSTEN, T. A.; ZHANG, X. Y.; KEHOE, P. Heightened cocaine and food self-administration in female rats with neonatal isolation experience. Neuropharmacology, Oxford, v.31, p.70-76, 2006.

KREIBICH, A. S.; BLENDY, J. A. cAMP response elementbinding protein is required for stress but not cocaineinduced reinstatement. J. Neurosci., Washington DC, v.24, p.6686-6692, 2004.

KUCZENSKI, R.; SEGAL, D. S.; AIZENSTEIN, M. L. Amphetamine, cocaine, and fencamfamine: relationship between locomotor and stereotypy response profiles and caudate and accumbens dopamine dynamics. J. Neurosci., Washington, v.11, p.2703-2712, 1991.

LEPORE, M.; VOREL, S. R.; LOWINSON, J.; GARDERNER, E. L. Conditioned place preference induced by delta-9-tetrahydrocannabinol: comparison with cocaine, morphine and food reward. Life Sci.,Oxford, v.56, p.2073-2080, 1995.

LEPSCH, L.; GONZALO, L.; MAGRO, F.; DELUCIA, R.; SCAVONE, C.; PLANETA, C. Exposure to chronic stress increases the locomotor response to cocaine and the basal levels of corticosterone in adolescent rats. Addict. Biol., Mannheim, v.10, p. 251-256, 2005.

LU, L.; GRIMM, J. W.; SHAHAM, Y.; HOPE, B. Molecular neuroadaptations in the accumbens and ventral tegmental area during the first 90 days of forced abstinence from cocaine self-administration.J. Neurochem., Oxford, v.85, p.1604-1613, 2003.
MARIN, M. T.; PLANETA, C. S. Maternal separation affects cocaine-induced locomotion and response to novelty in adolescent but not in adult rats. Brain Res., Amsterdam, v. 1013, p. 83-90, 2004.

McFARLAND, K.; DAVIDGE, S. B.; LAPISH, C. C.; KALIVAS, P. W. Limbic and motor circuitry underlying footshock-induced reinstatement of cocaine-seeking behavior. J. Neurosci., Washington, v.24, p.1551-1560, 2004.

MICZEK, K. A.; MUTSCHLER, N. H.; VAN ERP, A. M.; BLANK, A. D.; McINERNEY, S. C. d-amphetamine "cue" generalizes to social defeat stress: behavioral sensitization and attenuated accumbens dopamine. Psychopharmacology, Berlin, v.147, p.190-199, 1999.

MISERENDINO, M. J.; NESTLER, E. J. Behavioral sensitization to cocaine: modulation by the cyclic AMP system in the nucleus accumbens. Brain Res., Amsterdam, v.674, p.299-306, 1995.

MOFFETT, M. C.; HARLEY, J.; FRANCIS, D.; SANGHANI, S. P.; DAVIS WI.; KUHAR, M. J. Maternal separation and handling affects cocaine selfadministration in both the treated pups as adults and the dams. J. Pharmacol. Exp. Ther., Bethesda, v.3173, p.1210-1218, 2006.

MOFFETT, M. C.; VICENT, A.; KOZEL, M.; PLOSKY, P.; FRANCIS, D. D.; KUHAR, M. J. Maternal separation alters drug intake patterns in adulthood in rats. Biochem. Pharmacol., Oxford, v. 73, p. 321-330, 2007.

MUELLER, D.; STEWART, J. Cocaine-induced place preference: reistatement by priming injections of cocaine. Behav. Brain Res., Amsterdam, v.115, p.39-47, 2000.

NESTLER, E. J.; HYMAN S. E.; ROBERT, C. M. Molecular neuropharmacology: a foundation for clinical neuroscience. New York: McGraw-Hill, 2001. 539p.

O'BRIEN, C. P. Drug addiction and drug abuse. In: Brunton L. L.; Lazo, J. S.; Parker, K. L. (Eds.). Goodman and Gilman's the pharmacological basis of therapeutics. 11.ed. New York: Pergamon Press, 2005. p.607-627.

O'BRIEN, C. P.; EHRMAN, R. N.; TERNES, J. W. Classical conditioning in human opioid dependence. In: GOLDBERG, S. R., STOLERMAN, I. P. (Eds.) Behavioral analysis of drug dependence. Orlando: Academic Press, 1986. p.329-356. 
PHILliPS, T. J.; ROBERBS, A. J.; LESSOV, C. N. Behavioral sensitization to ethanol: genetics and the effects of stress. Pharmacol. Biochem. Behav., New York, v.57, p. 487-493, 1997.

PIAZZA, P. V.; DEMINIERE, J. M.; LE MOAL, M.; SIMON, H. Stress- and pharmacologically-induced behavioral sensitization increases vulnerability to acquisition of amphetamine self-administration. Brain Res., Amsterdam, v. 514, p. 22-26, 1990.

PICKENS, R.; THOMPSON, T. Cocaine-reinforced behavior in rats: effects of reinforcement magnitude and fixed-ratio size. J. Pharmacol. Exp. Ther., Bethesda, v.161, p.122-129, 1968.

PLANETA, C. S.; AIZENSTEIN, M. L.; DeLUCIA, R. Reinforcing properties of fencamfamine: involvement of dopamine and opioid receptor. Pharmacol. Biochem. Behav., Oxford, v.50, p.35-40, 1995a.

PLANETA, C. S.; DeLUCIA, R. AIZENSTEIN, M. L. The behavioral sensitization induced by fencamfamine is not related to plasma drug levels. Braz. J. Med. Biol. Res., São Paulo, v.28, p.667-670, 1995b.

PONTIERE, F. E.; TANDA, G.; DI CHIARA, G. Intravenous cocaine, morphine, and amphetamine preferentially increase extracellular dopamine in the "shell" as compared with "core" of the rat nucleus accumbens. Proc. Natl. Acad. Sci. USA, Washington, v.92, p.12304-12308, 1996.

PONTIERE, F. E.; TANDA, G.; ORZI, F.; DI CHIARA, G. Effects of nicotine on the nucleus accumbens and similarity to those of addictive drugs. Nature, New York, v.382, p.255-257, 1995.

POST, R. M.; CONTEL, N. R. Human and animal studies of cocaine: implications of development of behavioral pathology. In: CREESE, I. (Ed.). Stimulants: neurochemical, behavioral and clinical perspectives. New York: Raven, 1983. p. 169-203.

REID, M. S.; HO, L. B.; TOLLIVER, B. K.; WOLKOWITZ, O. M.; BERGER, S. P. Partial reversal of stress-induced behavioral sensitization to amphetamine following metyrapone treatment. Brain Res., Amsterdam, v.783, p.133-142, 1998.
ROBINSON, T. E.; BECKER, J. B. Enduring changes in brain and behavior produced by chronic amphetamine administration: a review and evaluation of animal models of amphetamine psychosis. Brain Res. Rev., Amsterdam, v.396, p.157-198, 1986.

ROBINSON, T. E.; BERRIDGE, K. C. Incentivesensitization and addiction. Addiction, Abingdon, v.96, p.103-114, 2001.

ROBINSON, T. E.; BERRIDGE, K. C. The neural basis of drug craving: an incentive-sensitization theory of addiction. Brain Res. Rev., Amsterdam, v.18, p.247-291, 1993.

ROBINSON, T. E.; BERRIDGE, K. C. The psychology and neurobiology of addiction: an incentive-sensitization view. Addiction, Abingdon, v.95, suppl2, p.S91-117, 2000.

SANCHEZ, C. J.; SORG, B. A. Conditioned fear stimuli reinstate cocaine-induced conditioned place preference. Brain Res., Amsterdam, v. 908, p. 86-92, 2001

SEGAL, D. S.; JANOWSKY, D. S. Psychostimulant-induced behavioral effects: possible models of schizophrenia. In: LIPTON, M. A.; DIMASCIO, A.; KILLAN, K. F.(Eds.). Psychopharmacology: a generation of progress. New York: Raven Press, 1978. p. 1113-1123.

SELYE, H. Confusion and controversy in the stress field. $J$. Hum. Stress, Washington, v.1, p.37-44, 1975.

SHIM, I.; JAVAID, J. I.; WIRTSHAFTER, D. JANG, S. Y.; SHIN, K. H.; LEE, H. J.; CHUNG, Y. C.; CHUN, B. G. Nicotine-induced behavioral sensitization is associated with extracellular dopamine release and expression of cFOS in the striatum and nucleus accumbens of the rat. Behav. Brain Res., Amsterdam, v.121, p.137-147, 2001.

SHIPPENBERG, T. S.; EMMETT-OGLESBY, M. W.; HERZ, A. Morphine-induced place preference is not confounded by drug-induced alterations in locomotor activity. Pharmacol. Biochem. Behav., New York, v.32, p.129-32, 1989.

SINHA, R. How does stress increase risk of drug abuse and relapse? Psychopharmacology, Berlin, v.158, p.343-359, 2001. 
SINHA, R.; CATAPANO, D.; O’MALLEY, S. Stressinduced craving and stress response in cocaine dependent individuals. Psychopharmacology, Berlin, v.142, p.343351, 1999.

SPEAR, L. P. The adolescent brain and age-related behavioral manifestations. Neurosci. Biobehav. Rev., Oxford, v.24, p.417-463, 2000.

SPYRAKI, C.; FIBIGER, H. C.; PHILlIPS, A. G. Dopaminergic substrates of amphetamine-induced place preference conditioning. Brain Res., Amsterdam, v.253, p.185-193, 1982.

STRECH, R.; GERBER, G. J.; WOOD, S. M. Factors affecting behavior maintained by response-contingent intravenous infusions of amphetamine in squirrel monkeys. Can. J. Physiol. Pharmacol., Ottawa, v. 49, p.581-589, 1971.

TANDA, G.; MUNZAR, P.; GOLDBERG, S. R. Selfadministration behavior is maintained by the psychoactive ingredient of marijuana in squirrel monkeys. Nature Neurosci., New York, v.3, p.1073-1074, 2000.

TANDA, G., PONTIERI, F. E., DI CHIARA, G. Cannabinoid and heroin activation of mesolimbic dopamine transmission by a common mul opioid receptor mechanism. Science, Washington DC, v.276, p.20482050, 1997.

THADANI, P. V. The intersection, of stress drug abuse and development. Psychoneuroendocrinology, Oxford, v.27, p.221-230, 2002.

TIDEY, J. W.; MICZEK, K. A. Acquisition of cocaine selfadministration after social stress: role of accumbens dopamine. Psychopharmacology, Berlin, v.130, p.203212, 1997.

TORNATZKY, W.; MICZEK, K. A. Cocaine selfadministration "binges": transition from behavioral and autonomic regulation toward homeostatic dysregulation in rats. Psychopharmacology, Berlin, v.148, p.289-298, 2000.
VEZINA, P.; QUEEN, A. L. Induction of locomotor sensitization by amphetamine requires the activation of NMDA receptors in the rat ventral tegmental area. Psychopharmacology, Berlin, v.151, p.184-91, 2000.

WALLACE, B. C. Psychological and environmental determinants of relapse in crack cocaine smokers. J. Subst. Abuse Treat., New York, v.6, p.95-106, 1989.

WARNER, L. A.; KESSLER, R. C.; HUGHES, M., ANTHONY, J. C.; NELSON, C. B. Prevalence and correlates of drug use and dependence in the United States. Results from the National Comorbidity Survey. Arch. Gen. Psychiatry, Chicago, v.52, p.219-229, 1995

WEISS, F. Neurobiology of craving, conditioned reward and relapse. Curr. Opinion Pharmacol., Oxford, v.5, p.9-19, 2005.

WISE, R. A.; BOZARTH, M. A. A psychomotor stimulant theory of addiction. Psychol. Rev., Washington, v.94, p.469-492, 1987.

WIT, H.; STEWART, J. Reinstatement of cocaine-reinforced responding in the rat. Psychopharmacology, Berlin, v.71, p.134-143, 1981.

WORLD HEALTH ORGANIZATION. International Statistical classification of diseases and related health problems. Geneve: WHO, 1992. 1243p.

ZHANG, X. Y.; SANCHEZ, H.; KEHOE, P.; KOSTEN, T. A. Neonatal isolation enhances maintenance but not reinstatement of cocaine self-administration in adult male rats. Psychopharmacology, Berlin, v.177, p.391-399, 2005.

Recebido para publicação em 29 de novembro de 2006. Aceito para publicação em 20 de junho de 2007. 